\title{
Coronary flow and reactivity, but not arrhythmia vulnerability, are affected by cardioplegia during cardiopulmonary bypass in piglets
}

Petru Liuba ${ }^{1 *}$, Sune Johansson ${ }^{2}$, Erkki Pesonen ${ }^{1}$, Michal Odermarsky $^{1}$, Axel Kornerup-Hansen ${ }^{5}$, Anders Forslid ${ }^{4}$, Elhadi H Aburawi ${ }^{6}$, Thomas Higgins', Malene Birck ${ }^{5}$ and Valeria Perez-de-Sa ${ }^{3}$

\begin{abstract}
Background: Surgery under cardiopulmonary bypass (CPB) is still associated with significant cardiovascular morbidity in both pediatric and adult patients but the mechanisms are not fully understood. Abnormalities in coronary flow and function have been suggested to play an important role. Prior studies suggest protective effects on coronary and myocardial function by short intravenous (i.v.) infusion of cyclosporine A before CPB.

Methods: Barrier-bred piglets (10-12 kg, n=20) underwent CPB for 45 min, with or without antegrade administration of cardioplegic solution. Prior to CPB, half of the animals in each group received an i.v. infusion of $100 \mathrm{mg} / \mathrm{kg}$ cyclosporine A. The left anterior descending coronary flow velocity responses to adenosine, serotonin, and atrial pacing, as well as left ventricular function and postsurgical vulnerability to atrial fibrillation (Afib) were assessed by intracoronary Doppler, epicardial echocardiography, and in vivo electrophysiological study, before and 8 hours after surgery. Plasma C-reactive protein (CRP) and fibrinogen were measured at both time-points.

Results: Cyclosporine infusion did not influence any of the studied variables $(p>0.4)$. Coronary peak flow velocity ( $C P F V$ ) rose significantly after surgery especially in the cardioplegia group $(p<0.01$ vs. non-cardioplegia group and pre-surgery). CPFV responses to adenosine, but not to serotonin, tended to decrease $(p=0.06)$ after surgery only in cardioplegia group ( $\mathrm{p}=0.06 ; \mathrm{p}=0.8$ in non-cardioplegia group vs pre-surgery). Also, cPFV response to atrial pacing was lower in the cardioplegia than in the non-cardioplegia group $(p=0.02)$. Neither vulnerability nor duration of induced Afib after CPB differed between groups (Chi-square $\mathrm{p}=0.4$ ). Cyclosporine had no significant effect on coronary indexes or arrhythmia vulnerability ( $p>0.4)$. There was no difference in systolic myocardial function between groups at any time point.

Conclusion: In piglets, CPB with cardioplegia was associated with profound abnormalities in coronary vasomotor tone and receptor-related flow regulation, whereas arrhythmia vulnerability appeared to be comparable with that in non-cardioplegia group. In this study, preconditioning with cyclosporine had no detectable protective effect on coronary circulation or arrhythmia vulnerability after CPB.
\end{abstract}

Keywords: Cardiopulmonary bypass, Coronary, Cardioplegia, Piglets

\footnotetext{
* Correspondence: petru.liuba@med.lu.se

'Division of Cardiology, Children's Heart Center, Skåne University Hospital, Lund, Sweden
}

Full list of author information is available at the end of the article 


\section{Background}

The survival and quality of life of patients with congenital heart disease (CHD) undergoing surgery with cardiopulmonary bypass (CPB), even with very complex cardiac malformations, has improved significantly during the past decade. Nevertheless, surgery with CPB remains associated with appreciable cardiovascular morbidity in both pediatric and adult patients [1]. Recent clinical studies have highlighted several modifiable and nonmodifiable risk factors which could affect the cardiac function; intraoperative variables such as duration of $\mathrm{CPB}$ and aortic cross-clamping, type and temperature of cardioplegic solution seem to significantly influence the postoperative outcome [1,2]. Although the mechanisms whereby these different factors, together or independently, influence cardiovascular function remain unclear, perioperative changes in coronary flow and function might be a common pathway [3].

A prior study suggested that coronary vasomotor function is altered by cold crystalloid cardioplegia [4], but to a less extent by warm blood cardioplegia [5]. These changes appear to involve both the microvascular [6] and epicardial [7] coronary bed with significant coronary endothelial damage and apoptosis. A study by Oka et al. [8] suggested that addition of cyclosporine $\mathrm{A}$ to the cardioplegic solution could alleviate cardiomyocyte's mytochondrial dysfunction and hence protect the myocardium against CPB-related ischemic injury.

We have earlier demonstrated that coronary flow is altered after surgery with $\mathrm{CPB}$ in children with CHD [9]. The observation of increased coronary blood flow after surgery with $\mathrm{CPB}$, particularly in the setting of impaired coronary vasomotor function, should intuitively suggest decreased coronary flow reserve, which has been speculated to contribute to myocardial dysfunction and arrhythmias postoperatively [10]. To our knowledge, the relationship between coronary vasomotor function and arrhythmia vulnerability in the early postoperative period has not yet been investigated.

In the present study, we investigated whether administration of normothermic blood cardioplegia influence coronary and myocardial function after $\mathrm{CPB}$ in young miniaturized pigs, and whether these changes could be prevented by preoperative intravenous infusion of cyclosporine A. The threshold of postoperative tachyarrhythmia was also studied.

\section{Methods}

Twenty barrier-bred, specific-pathogen-free, 8-9-week old, Göttingen minipigs (Ellegaard Göttingen Minipigs, Dalmose, Denmark), housed in a barrier-protected animal facility under controlled conditions (temperature between 18 and $22^{\circ} \mathrm{C}$ and relative humidity 30-60\%) were allowed to acclimatize for 1 week before the start of the study. All animals received proper care in compliance with the Swedish Ethics Guidelines for the use of laboratory animals.

On the study day, the animals were randomized to one of the study groups and sedated with a mixture of ketamin and midazolam $(10 \mathrm{mg} / \mathrm{kg}$ and $1 \mathrm{mg} / \mathrm{kg}$, respectively) given intramuscularly before a $22 \mathrm{G}$ cannula was inserted into an ear vein. Under sedation with propofol i.v. (approximately $0.5-2 \mathrm{mg} / \mathrm{kg}$ ), the animals were orally intubated with a cuffed endotracheal tube (size 3.5 - 4.5). Mechanical ventilation was then started with oxygenenriched room air (air and pure $\mathrm{O}_{2}$ in ratio of $2.2: 1 ; 8 \mathrm{~mL} / \mathrm{kg}$, 30 breaths/min, 5 PEEP) using a Siemens SERVO 300 respirator (Siemens, Erlangen, Germany).

The animals were subsequently placed prone on an $\mathrm{X}$-ray table and monitored with pulse oximetry (\% $\mathrm{Sp}_{\mathrm{O}}$ ) and a 3-lead EKG. Central temperature was monitored by a rectal probe. Through surgical cutdown, a $5 \mathrm{~F}$ double-lumen central venous catheter (COOK Medical Inc, IN, USA) was inserted into the left internal jugular vein for drug administration and central venous pressure monitoring, and a $20 \mathrm{G}$ cannula was inserted into the left carotid artery for blood pressure monitoring and blood sampling. A baseline blood sample was taken and preserved in ice for C-reactive protein, plasma lipids and fibrinogen analysis. Intracoronary doppler velocimetry was thereafter performed according to the protocol described below.

Following coronary assessment, the animals received for 10 minutes an intravenous infusion of either cyclosporine A $100 \mathrm{mg} / \mathrm{kg}$ or saline via the central venous line. Cardiac rhythm monitoring with a portable Holter system was performed for 5 minutes preoperatively and continued immediately after surgery until the end of the postoperative coronary assessment (8 hours after surgery).

\section{Coronary velocimetry protocol}

The coronary flow velocity study was performed before and 6-8 hours after completion of surgery $[11,12]$. After an intravenous bolus of $100 \mathrm{IU} / \mathrm{kg}$ heparin, a $4 \mathrm{~F}$ short sheath was inserted into the right carotid artery and a $4 \mathrm{~F}$ Judkins right coronary catheter was advanced into the ostium of the left coronary artery (LCA) under fluoroscopy guidance. A Volcano ComboWire (Pressure/Flow Guide .014" Wire, Volcano, CA, USA) connected to a real-time spectrum analyzer (ComboMap System, model 6800, Volcano, CA, USA), was then inserted through the catheter and positioned in the mid-part of the left anterior descending coronary artery (LAD). Coronary averaged peak velocity (APV) and pressure were displayed continuously on the system's monitor. Data were stored on the hard disk for off-line analysis. After stabilization of the baseline Doppler signal, intracoronary adenosine $(5 \mu \mathrm{g} / \mathrm{kg})$, bradykinin $\left(10^{-6} \mathrm{M}\right)$, and serotonin $\left(10^{-6} \mathrm{M}\right)$ 
were each given as a bolus through the catheter and the peak averaged velocity assessed. All drugs were given in succession 10 min apart, and in the same order, in all animals. Complete recovery of coronary flow velocity and pressure (ie, steady state) was attained in each animal before the next drug was given.

Before administration, all drugs were diluted with $0.9 \%$ saline to a volume of $3 \mathrm{ml}$. A recovery period of $10 \mathrm{~min}$ was allowed between drug administration until the coronary flow velocity values returned to baseline.

Postoperatively, coronary velocity response to incremental increases in heart rate ( 20 beats/minute every 2 minutes up to 60 beats/minute over the baseline heart rate) was assessed as well.

\section{Echocardiography}

Epicardial echocardiography was performed before the start of CPB (after thoracotomy) and 6-8 hours after $\mathrm{CPB}$, immediately before the postoperative coronary flow study. A Philips ultrasound system (Royal Philips Electronics, Amsterdam, The Netherlands) equipped with a $7-\mathrm{MHz}$ probe was used. Standard 4- and 5chamber apical and short-axis parasternal views were obtained in all animals. Left ventricle's (LV) and aortic valve dimensions, aortic outflow signal and mitral inflow signal were assessed by Doppler as earlier described [13]. All images were stored on the system's hard disk for later analysis of LV area and volume, systolic function (ejection fraction and shortening fraction), and diastolic function (mitral E/A ratio, total and "E"/"A"VTI).

\section{CPB}

Through a median sternotomy, the pericardium was opened, and the aorta and caval veins cannulated after heparinization $(300 \mathrm{U} / \mathrm{kg})$. CPB, using a centrifugal pump (Bio-Medicus, Medtronic USA inc., MN, USA) and a pediatric membrane oxygenator (Minimax Plus, Medtronic USA inc., MN, USA), was instituted with a flow rate of $2.5-3 \mathrm{~L} / \mathrm{min} / \mathrm{m} 2$, and the animals were systemically cooled to 32 degrees Celsius. The system was primed with $500 \mathrm{~mL}$ of whole blood stabilized with citrate (Veterinary Pharmacy, University of Copenhagen, Denmark) sampled 3-6 days prior to surgery from slaughter pigs anaesthetized with isoflurane (Baxter, Allerød, Denmark). The hematocrit was maintained between $25 \%$ to $30 \%$, and the mean arterial pressure around $60 \mathrm{~mm} \mathrm{Hg}$. CPB was carried out for $45 \mathrm{~min}$, either without $(\mathrm{n}=10)$ or with antegrade administration for 20 minutes of blood cardioplegia $(n=10)$. The ascending aorta was thereafter cross-clamped, and a double-lumen aortic root cannula inserted for antegrade delivery of cardioplegic solution. Cardioplegia was given via a roller pump that mixed oxygenated blood with modified St Thomas' cardioplegic solution in a $4: 1$ ratio $(30 \mathrm{ml} / \mathrm{kg})$, at a pressure of ca $60 \mathrm{~mm} \mathrm{Hg}$. The cardioplegic solution was kept at room temperature before delivery. During weaning from $\mathrm{CPB}$, all animals were started on dopamine $5 \mu \mathrm{g} / \mathrm{kg} / \mathrm{min}$, milrinone $0.5 \mu \mathrm{g} / \mathrm{kg} / \mathrm{min}$ (maintenance dose), and $5 \%$ albumin $(50 \mathrm{mg} / \mathrm{ml}$, CSL Behring, Danderyd, Sweden) $25 \mathrm{ml} /$ hour in order to maintain stable hemodynamics. Epicardial electrodes were placed in standard position ( 2 on the right atrium and 2 on the right ventricle) for later cardiac pacing during coronary velocity assessment.

\section{Statistics}

All coronary responses to vasoactive agonists and cardiac pacing are expressed as a ratio of peak (after drug infusion) to baseline (before drug infusion) velocities (peak-to-baseline). Significant differences between groups in numerical variables were calculated with ANOVA followed, when indicated, by Bonferroni posthoc test. To reduce the impact of scattering and skewness, given the small number of animals, all numerical variables were log-transformed. Differences were considered statistically significant at $P<0.05$. Statistical analysis was carried out with SAS 9.1 (SAS Institute, Cary, $\mathrm{NC}$ ). Data are presented as means and SD. Mean arterial pressure and blood gases $\left(\mathrm{O}_{2}\right.$ and $\left.\mathrm{CO}_{2}\right)$ were maintained within normal range in all animals postoperatively.

\section{Results}

Two animals died postoperatively, one from the noncardioplegia\&cyclosporine group due to ventricular fibrillation (no resuscitation attempted) and the other one from the cardioplegia \& non-cyclosporine group due to recurrent massive bleeding. All data from these animals were therefore discarded.

\section{Cyclosporine on post-CPB variables}

Cyclosporine had no significant effect on coronary indexes or arrhythmia vulnerability $(p>0.4)$. Neither inflammatory or lipid variables were influenced by cyclosporine $(\mathrm{p}>0.5)$.

There were no significant differences in MAP or blood gases between treatment groups $(\mathrm{p}>0.4)$. All animals were in sinus rhythm after CPB. Holter analysis during the postoperative period revealed short-lasting, hemodynamycally insignificant episodes of atrial-ventricular block II, supraventricular tachycardia and atrial fibrillation/flutter (AFib/AF) in nearly all animals. The frequency and duration of AFib/AF were similar in all groups ( $\mathrm{p}>0.2)$.

\section{Inflammatory and lipid data}

Neither C-reactive protein (CRP) nor fibrinogen concentrations differed between the groups at any timepoint ( $>0.05)$. Among lipid variables, HDL dropped significantly 
after CPB in the cardioplegia group $(\mathrm{p}=0.03)$ but remained relatively unchanged in the noncardiopegia group $(\mathrm{p}>0.1)$. Cyclosporine had no influence on any of these variables $(\mathrm{p}>0.2)$.

\section{Coronary peak velocity assessment}

Coronary peak flow velocity (cPFV) rose significantly after surgery in both cardioplegia and noncardioplegia animals ( $<<0.01$ vs. pre-surgery for both groups), with the largest velocity increase in the cardioplegia group ( $\mathrm{p}=0.001$ vs noncardioplegia group; Figure 1). cPFV responses to adenosine, but not to serotonin ( $>0.2)$, tended to decrease after surgery only in the cardioplegia group $(\mathrm{p}=0.06)$. In contrast, cPFV response to atrial pacing was lower in cardioplegia than in non-cardioplegia animals ( $\mathrm{p}=0.02$; Figure 2 ).

\section{Arrhythmia threshold}

Afib was induced in all animals postoperatively at a threshold of 180-200 msec. However, neither this nor the duration of induced Afib differed between groups $(\mathrm{p}=0.4)$.

\section{Epicardial echocardiography data}

LV end-diastolic diameters were significantly larger after $\mathrm{CPB}(\mathrm{p}=0.03)$, whereas postoperative EF and SF were comparable to preoperative values ( $p>0.2)$. Similarly, $\mathrm{CO}$ and mitral inflow variables (E/A, total velocity time integral (VTI), and VTI"E"/VTI"A") remained unchanged as compared to preoperative values $(\mathrm{p}>0.1)$.

\section{Discussion}

Using reproducible and sensitive in-vivo methods for evaluation of coronary vasomotor and cardiac function $[13,14]$, we aimed to assess whether the use of tepid

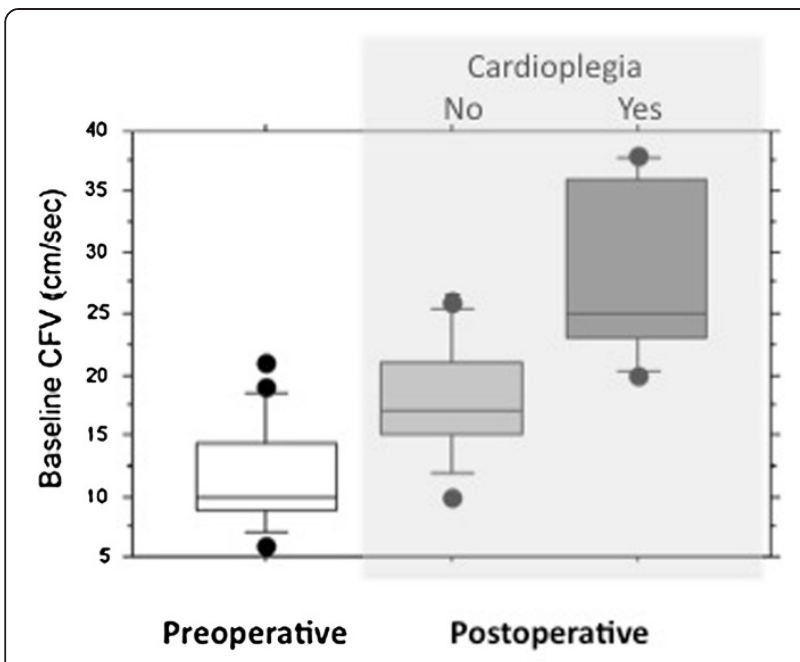

Figure 1 Baseline averaged coronary flow velocities in animals before (empty bar), and after surgery with (light gray bar) and without cardioplegia (dark gray bar).

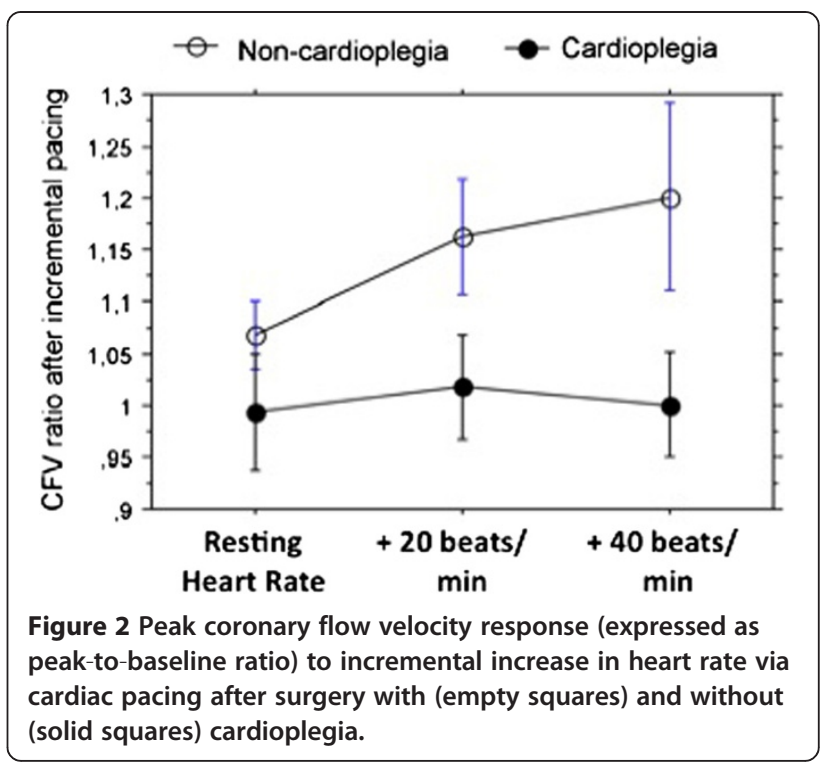

blood cardioplegia during $\mathrm{CPB}$ of miniature piglets would affect the heart and the coronaries during the first hours after surgery, and whether preconditioning with cyclosporine A could alleviate the putative negative impact of cardioplegia on these indexes. Inflammatory and lipid indexes were also assessed. Using several vasoactive agonists known to dilate the coronaries through separate molecular endothelium-dependent and independent mechanisms, we observed cardioplegia-related abnormalities in coronary vasomotor tone and reactivity 6 to 8 hours post-CPB, though both the inflammatory markers (C-reactive protein and fibrinogen) and the myocardial function (assessed by epicardial standard echocardiography) were comparable to preoperative values. Furthermore, blood cardioplegia did not seem to influence in any way the susceptibility to postoperative arrhythmias, as demonstrated by the equal frequency of spontaneous and stimulator-induced rhythm disturbances in the cardioplegia and non-cardioplegia groups. No significant preconditioning effect of cyclosporine on coronary function was demonstrated. In the present study, the coronary flow measurements were achieved using the Volcano Combomap system, which has the advantage of assessing simultaneously both pressure and flow velocity (Figure 3/Panel A) via a 0.014 " coronary wire (Figure 3/Panel B). Our group has a certain expertise with using this device in the swine model $[11,12]$.

Cardiopulmonary bypass (CPB) remains a central part of surgery of congenital heart defects in both pediatric and adult patients [1]. In spite of major technical improvements in the performance of CPB during the past two decades, cardiovascular complications, particularly during the early postoperative period, are still present. While postoperative tachyarhythmias, often in form of atrial fibrillation (AFib), are more common in adults, 

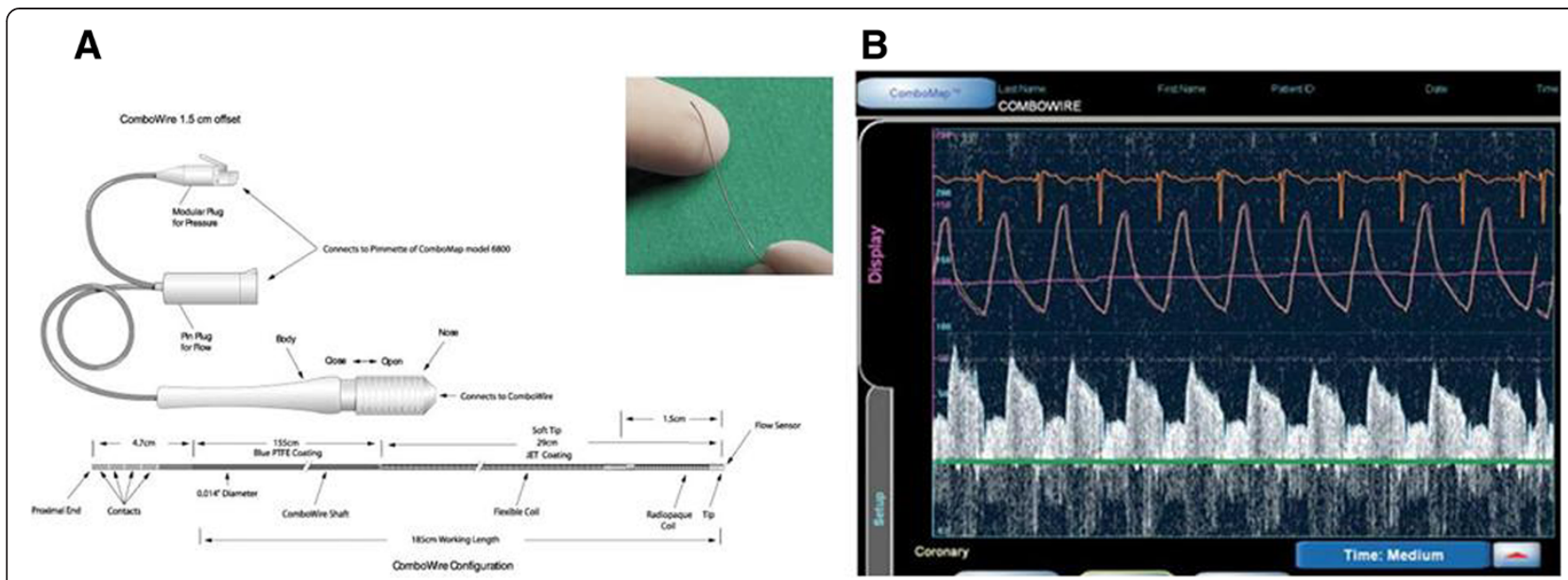

Figure 3 Illustration of Volcano ComboMap system and Combowire. Panel A: Volcano ComboMap ${ }^{\circledR}$ screenshot showing both pressure and flow measurements in coronary circulation; Panel B: illustration of Combowire used for intracoronary flow and pressure assessment.

heart failure and vascular complications, such as pulmonary and systemic hypertension, appear to be less agerelated, with a greater prevalence among patients with depressed cardiac function at the time of surgery $[1,2,15]$. Prior in-vitro studies have provided suggestive evidence that some of these complications might relate to profound coronary and systemic vascular dysfunction that occurs during reperfusion [4-7]; others $[8,16]$ have suggested potential benefit in partially reversing these effects by substances with protecting effects of vascular endothelium and cardiomyocites (pre- or postconditioning).

The increase in coronary flow after cardiac surgery has been demonstrated both in animal and clinical studies $[9,17]$, but the replication of this phenomenon in the present study is a key finding, since tepid blood was used instead of cold crystalloid cardioplegia. The mechanisms might relate to elevated circulating levels of cytokines (not measured in this study), in particular tumor necrosis factor-alpha (TNF- $\alpha$ ), and complement activation [18], which are triggered mainly through the contact between the blood and the foreign surface of the CPB circuit. TNF$\alpha$ upregulates inducible nitric oxide $(\mathrm{NO})$ synthase activity with secondary increased release of NO, thereby contributing to the reduction in the intrinsic tone of the coronary microcirculation [19]. Increased amounts of intravascular NO lead to formation of peroxynitrate, which is highly damaging for endothelial cells [20]. This effect is further exacerbated by ischemia-reperfusion injury [21]. The involvement of the coronary endothelium is also suggested by the difference in coronary velocity response to cardiac pacing, which is known to cause dilatation through the release of endothelial NO [22,23]. Dysfunction of coronary smooth muscle cells might occur as well, as indicated by the mildly impaired coronary response to adenosine.

In the present study, preconditioning with cyclosporine A prior to $\mathrm{CPB}$ conferred no observable protection on either coronary flow or function postoperatively. Although, in high doses, ie, following cardiac transplantation as an immunosuppressive agent, cyclosporine is known to deter coronary endothelial function [24], when used in lower doses for short periods of time or as addition to the cardioplegia solution, it appears to exert favourable effects on both the cardiomyocytes and coronary vascular function $[8,25]$. Cyclosporine A has been reported to ameliorate reperfusion injury in several in vitro and in vivo animal models of unprotected and protected ischemia [26,27]. In a neonatal piglet model of cardioplegic arrest [8], cyclosporine A was shown to prevent apoptosis-related mytocondrial dysfunction. Similar effect of cyclosporine A on mitochondria was demonstrated in a rabbit model of ischemia-reperfusion, and was associated with improved post-cardioplegia myocardial performance [28]. Therefore we decided to test its eventual efficacy in protecting the coronary function in this animal model. The lack of effect in the present study could be explained in part by the relatively mild coronary dysfunction, which, perhaps in conjunction with the postoperative use of milrinone [29], another drug with important vasoprotective action, might have concealed a potential effect of cyclosporine. The preserved postoperative myocardial function, documented by echocardiography, could support this assumption.

\section{Conclusions}

In conclusion, cardioplegia with tepid blood during surgery with $\mathrm{CPB}$ in piglets was associated with abnormalities in coronary vasomotor tone and receptor-related flow regulation, whereas myocardial function appeared to be preserved. In this study, preoperative administration of cyclosporine had no observable protective effect on coronary circulation or arrhythmia vulnerability after $\mathrm{CPB}$. 


\section{Competing interests}

The authors declare that they have no competing interests.

\section{Authors' contributions}

PL designed the study, performed the coronary Doppler study, contributed to the statistical analysis, and drafted the manuscript. SJ performed the cardiac surgery. EP contributed to the coronary Doppler study. MO participated in the analysis of the coronary and heart rhythm data. $\mathrm{TH}$ and EA performed the cardiac ultrasound study. VP, AKH, AF, and MB were responsible for sedation and anaesthesia, and the periperative care of animals. All authors read and approved the final manuscript.

\section{Acknowledgements}

We are most indebted for the very kind support and cooperation of Dr Jens Johansson, pediatric cardiac surgeon, Dr Eric Johansson, and perfusionists Ann-Katrin Krokström and Thomas Hansson. We are also most grateful to Annica Maxedius, research nurse, for invaluable technical assistance throughout the study. The study was supported via grants from Lund University Hospital, and the Swedish Heart-Lung Foundation.

\section{Author details}

${ }^{1}$ Division of Cardiology, Children's Heart Center, Skåne University Hospital, Lund, Sweden. 'Division of Cardiac Surgery, Children's Heart Center, Skåne University Hospital, Lund, Sweden. ${ }^{3}$ Division of Anesthesiology and Intensive Care, Children's Heart Center, Skåne University Hospital, Lund, Sweden. ${ }^{4}$ Department of Animal Science, Lund University, 22185 Lund, Sweden. ${ }^{5}$ Department of Veterinary Disease Biology, Faculty of Life Sciences, University of Copenhagen, Copenhagen, Denmark. ${ }^{6}$ Department of Paediatrics, College of Medicine and Health Sciences, UAE University, AI Ain, UAE.

Received: 8 January 2013 Accepted: 4 June 2013

Published: 19 June 2013

\section{References}

1. Abarbanell GL, Goldberg CS, Devaney EJ, Ohye RG, Bove EL, Charpie JR: Early surgical morbidity and mortality in adults with congenital heart disease: the University of Michigan experience. Congenit Heart Dis 2008, 3:82-89.

2. Yves $A G$, Louagie MD, Emmanuel Gonzalez MD, Jacques Jamart MDC, Brigitte Malhomme MS, Serge Broka MD, Michel Buche MD, Philippe M, Eucher MD, Jean-Claude Schoevaerdts MD: Assessment of Continuous Cold Blood Cardioplegia in Coronary Artery Bypass Grafting. Ann Thorac Surg 1997, 63:689-696.

3. Sellke FW, Friedman M, Dai HB, Shafique T, Schoen FJ, Weintraub RM, Johnson RG: Mechanism causing coronary microvascular dysfunction following crystalloid cardioplegia and reperfusion. Cardiovasc Res 1993, 27:1925-1932.

4. Li S, Lin J, Lenehan E, Liu J, Long C, Liu J, Geng YJ: Myocardial protection of warm cardioplegic induction on the isolated perfused rat heart model. J Extra Corpor Technol 2004, 36:58-65.

5. Chang CH, Lin PJ, Chu Y, Lee YS: Impaired endothelium-dependent relaxation after cardiac global ischemia and reperfusion: role of warm blood cardioplegia. J Am Coll Cardiol 1997, 29:681-687.

6. Long C, Hu X, Zhang J, Xiu R, Guan Y: Changes of microvascular vasomotion and oxygen metabolism during cooling and rewarming period of cardiopulmonary bypass. J Extra Corpor Technol 2003, 35:13-16.

7. Yeh CH, Wang YC, Wu YC, Chu JJ, Lin PJ: Continuous tepid blood cardioplegia can preserve coronary endothelium and ameliorate the occurrence of cardiomyocyte apoptosis. Chest 2003, 123:1647-1654.

8. Oka N, Wang L, Mi W, Zhu W, Honjo O, Caldarone CA: Cyclosporine A prevents apoptosis-related mitochondrial dysfunction after neonatal cardioplegic arrest. J Thorac Cardiovasc Surg 2008, 135:123-130.

9. Aburawi EH, Berg A, Liuba P, Pesonen E: Effects of cardiopulmonary bypass surgery on coronary flow in children assessed with transthoracic Doppler echocardiography. Am J Physiol Heart Circ Physiol 2007, 293:1138-1143.

10. Hoffman JIE: Maximal coronary flow and the concept of coronary vascular reserve. Circulation 1984, 70:153-159.

11. Liuba P, Pesonen E, Forslid A, Paakkari I, Kornerup-Hansen A, Kovanen P Pentikäinen $M$, Persson $K$, Østergård $G$ : Protective effects of simvastatin on coronary artery function in swine with acute infection. Atherosclerosis 2006, 186:331-336.
12. Birck MM, Pesonen E, Odermarsky M, Hansen AK, Persson K, Frikke-Schmidt $H$, Heegaard PM, Liuba P: Infection-induced coronary dysfunction and systemic inflammation in piglets are dampened in hypercholesterolemic milieu. Am J Physiol Heart Circ Physiol 2011, 300:H1595-H1601.

13. Reeves ST, Glas KE, Eltzschig H, Mathew JP, Rubenson DS, Hartman GS, Shernan SK: Council for Intraoperative Echocardiography of the American Society of Echocardiography; Society of Cardiovascular Anesthesiologists. Guidelines for performing a comprehensive epicardial echocardiography examination: recommendations of the American Society of Echocardiography and the Society of Cardiovascular Anesthesiologists. Anesth Analg 2007, 105:22-28.

14. Denardo SJ, Talbot L, Hargrave VK, Selfridge AR, Ports TA, Yock PG: Accuracy of Doppler catheter measurements: effect of inhomogeneous beam power distribution on mean and peak velocity. J Am Coll Cardiol 1997, 29:283-292.

15. Denault A, Deschamps A, Tardif JC, Lambert J, Perrault L: Pulmonary hypertension in cardiac surgery. Curr Cardiol Rev 2010, 6:1-14.

16. Okorie M, Bhavsar D, Ridout D, Charakida M, Deanfield J, Loukogeorgakis S, MacAllister R: Postconditioning protects against human endothelial ischaemia-reperfusion injury via subtype-specific $\mathrm{K}_{\text {ATP }}$ channel activation and is mimicked by inhibition of the mitochondrial permeability transition pore. Eur Heart J 2011, 32:1266-1274.

17. Oskarsson G, Pesonen E, Gudmundsson S, Ingimarsson J, Sandström S, Werner O: Coronary flow reserve in the newborn lamb: an intracoronary Doppler guide wire study. Pediatr Res 2004, 55:205-210.

18. Sellke FW, Boyle EM Jr, Verrier ED: Endothelial cell injury in cardiovascular surgery: the pathophysiology of vasomotor dysfunction. Ann Thorac Surg 1996, 62:1222-1228.

19. Ku DD: Coronary vascular reactivity after acute myocardial ischemia. Science 1982, 218:576-578.

20. Rubanyi GM, Vanhoutte PM: Superoxide anions and hyperoxia inactivate endothelium-derived relaxing factor. Am J Physiol Heart Circ Physiol 1986, 250:H822-H827.

21. Khan TA, Bianchi C, Ruel M, Voisine P, Li J, Liddicoat JR, Sellke FW: Mitogenactivated protein kinase inhibitor and cardioplegia-cardiopulmonary bypass reduce coronary myogenic tone. Circulation 2003, 108:348-353.

22. Tayama S, Okumura K, Matsunaga T, Tsunoda R, Tabuchi T, Iwasa A, Yasue $\mathrm{H}$ : Influence of chronic nitric oxide inhibition of coronary blood flow regulation: a study of the role of endogenous adenosine in anesthetized, open-chested dogs. Jpn Circ J 1998, 62:371-378.

23. Nishikawa Y, Ogawa S: Importance of nitric oxide in the coronary artery at rest and during pacing in humans. J Am Coll Cardiol 1997, 29:85-92.

24. Petrakopoulou P, Anthopoulou L, Muscholl M, Klauss V, von Scheidt W, Uberfuhr P, Meiser BM, Reichart B, Weis M: Coronary endothelial vasomotor function and vascular remodeling in heart transplant recipients randomized for tacrolimus or cyclosporine immunosuppression. J Am Coll Cardiol 2006, 47:1622-1629.

25. Duan X, Ji B, Yu K, Liu J, Hei F, Long C: Pharmacological postconditioning protects isolated rat hearts against ischemia-reperfusion injury: the role of mitochondrial permeability transition pore. ASAIO J 2011, 57:197-202.

26. Argaud L, Gateau-Roesch O, Muntean D, Chalabreysse L, Loufouat J, Robert $D$, et al: Specific inhibition of the mitochondrial permeability transition prevents lethal reperfusion injury. J Mol Cell Cardiol 2005, 38:367-374.

27. Nathan M, Friehs I, Choi YH, Cowan DB, Cao-Danh H, McGowan FX, et al: Cyclosporin A but not FK-506 protects against dopamine-induced apoptosis in the stunned heart. Ann Thorac Surg 2005, 79:1620-1626

28. Oka N, Wang L, Mi W, Caldarone CA: Inhibition of mitochondrial remodeling by cyclosporine A preserves myocardial performance in a neonatal rabbit model of cardioplegic arrest. J Thorac Cardiovasc Surg 2008, 135:585-593.

29. Sidi A, Muehlschlegel JD, Kirby DS, Lobato EB: Administration of milrinone before ischemia, in the presence of beta-blockade, to treat metabolic impairment and myocardial stunning in pigs. Acta Anaesthesio/ Scand 2008, 52:397-405.

\section{doi:10.1186/1749-8090-8-157}

Cite this article as: Liuba et al:: Coronary flow and reactivity, but not arrhythmia vulnerability, are affected by cardioplegia during cardiopulmonary bypass in piglets. Journal of Cardiothoracic Surgery 2013 8:157. 